\title{
Clozapine-Induced Gastrointestinal Hypomotility: A 22-Year Bi-National Pharmacovigilance Study of Serious or Fatal 'Slow Gut' Reactions, and Comparison with International Drug Safety Advice
}

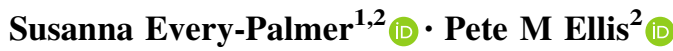

Published online: 16 June 2017

(c) The Author(s) 2017. This article is an open access publication

\begin{abstract}
Introduction Clozapine is the preferred antipsychotic for treatment-resistant schizophrenia, but has significant adverse effects, including gastrointestinal hypomotility or 'slow gut', which may result in severe constipation, ileus, bowel obstruction, and even death. These gastrointestinal effects remain inadequately recognized.

Methods We reviewed all reports of serious clozapine-induced gastrointestinal hypomotility (CIGH) submitted to the Australian Therapeutic Goods Administration and New Zealand Pharmacovigilance Centre between 1992 and 2013. We extracted relevant demographic, clinical, and outcome data and derived a numerator from clozapine registries. We examined whether clozapine drug safety information in Australia, New Zealand, the US, and the UK was adequate and consistent with pharmacoepidemiologic evidence.

Results A total of 43,132 people commenced clozapine over the study period. 160 were reported as having serious gastrointestinal hypomotility with clozapine the suspected cause (37/10,000 clozapine users). Of these, $66.3 \%$ were male, age range was $17-76$ years, clozapine dose range 25-1000 mg/day (mean $439 \mathrm{mg} /$ day) and median duration of clozapine treatment 2.5 years. Few had received laxatives. At least 29 patients died (7/10,000 clozapine users), a
\end{abstract}

Susanna Every-Palmer

susanna.every-palmer@ccdhb.org.nz

1 Te Korowai Whāriki Central Regional Forensic Service, Capital and Coast District Health Board, PO Box 50-233, Ratonga Rua-O-Porirua, Raiha Street, Porirua, Wellington, New Zealand

2 Department of Psychological Medicine, University of Otago, Wellington, PO Box 7343, Wellington 6242, New Zealand reported case fatality rate of $18 \%$. The CIGH prevalence, while similar to other smaller studies, differs significantly from clozapine prescribing information issued by regulators and pharmaceutical companies, none of which mention $\mathrm{CIGH}$, and which report serious gastrointestinal complications at rates of $<1 / 10,000$, almost a 40 -fold difference. Conclusion This is the largest study to date of serious CIGH. The reported prevalence of serious CIGH was $37 / 10,000$, a likely underestimation of true prevalence. Current prescribing guidelines provide inadequate information on CIGH. This may be contributing to poor awareness and high associated morbidity and mortality. It is time regulators and manufacturers update their guidance.

\section{Introduction}

Clozapine remains the 'gold standard' in treatment-resistant schizophrenia [1], with its superiority well established in terms of mental health outcomes, quality of life, and life expectancy [2-4]. However, clozapine's advantages come at a cost, with an array of problematic adverse effects of which clozapine-induced gastrointestinal hypomotility (CIGH) is one of the most serious [5, 6], albeit one that has received scant attention until the last decade.

CIGH is defined as an acquired state of delayed transit through the gastrointestinal tract (i.e. transit time $>2$ standard deviations [SD] above the mean), colloquially known as clozapine-related 'slow gut', resulting from the drug's pharmacological actions on the enteric nervous system [7]. In vitro, clozapine is shown to have potent effects on the mammalian colon, inhibiting neurogenic and, at higher concentrations, myogenic contractions, profoundly disrupting gastrointestinal motility [7]. These effects can be reversed by carbachol and to some extent by 
serotonin, suggesting both anticholinergic and anti-serotonergic mechanisms [7].

CIGH is very common. Between $50-80 \%$ of clozapinetreated patients have unambiguous objective evidence of CIGH in colonic transit studies [5, 6], with mean transit times that are four times longer than normal, and all regions of the colon affected [6]. While serious complications of CIGH such as ileus and pseudo-obstruction-the focus of this paper-attract the most attention, the less serious but highly prevalent manifestations, including dysphagia, dyspepsia, esophageal reflux, gastroparesis, and chronic constipation, affect quality of life for many clozapine users. For example, a systematic review of 32 studies calculated a pooled prevalence of clozapine-associated constipation of $31 \%$ [8], with chronic constipation consistently shown to significantly degrade quality of life for those it affects [9-11].

The US Food and Drug Administration (FDA) has mandated 'black box' warnings for clozapine, highlighting the risks of agranulocytosis, myocarditis, cardiomyopathy, seizures, and severe hypotension-but not CIGH. However, recent epidemiological studies suggest mortality related to CIGH $(10-12$ per $10,000[12,13])$ is at least 3 -fold that of the better-recognized complication of agranulocytosis [14].

Despite the prevalence and potential seriousness of CIGH, knowledge about preventing, identifying, and managing it remains poor, even among those most familiar with clozapine. In a 2016 study, only $50 \%$ of psychiatric inpatient nurses were aware clozapine could cause constipation [15]. In an Australian audit of 67 long-stay patients taking clozapine, there was a high level of baseline hematological and metabolic monitoring, but no monitoring of bowel function [16]. Of particular concern, clozapine treatment guidelines rarely mention the CIGH spectrum, let alone advise on its management [8, 17].

In this observational study, we examined all reports of suspected CIGH reactions received by the Australian and New Zealand pharmacovigilance agencies over a 22-year period (1992-2013 inclusive) to determine the demographics, risk factors, and outcomes related to serious or life-threatening $\mathrm{CIGH}$, and to calculate the proportion of clozapine-prescribed patients reported as having CIGHtype adverse drug reactions (ADRs). Australia and New Zealand both have high-quality pharmacovigilance programs monitoring all reported ADRs. At the time of data collection, New Zealand and Australia had the third and tenth highest rate per capita of ADR reporting (from 110 member countries) to the World Health Organization (WHO) global database, Vigibase [18]. Clozapine has particularly good pharmacovigilance data in New Zealand, being one of a handful of medications intensively scruti- nized by an Intensive Medicines Monitoring Program (IMMP) between 2000 and 2008, which tracked high-risk medicines in prospectively identified cohorts by prescription event monitoring [19]. At all other times in New Zealand clozapine was monitored by the Centre for Adverse Reactions Monitoring (CARM).

In both New Zealand and Australia, ADR reports are obtained through spontaneous reporting to the New Zealand Pharmacovigilance Centre and the Australian Therapeutic Goods Administration (TGA), respectively, by doctors, nurses, pharmacists, and pharmaceutical companies. All clinical events are assessed and coded by medical assessors using terms from the 'WHO Adverse Reaction Terminology' dictionary [19]. Causality assessment is performed for each event to determine the relationship with the medicine [20].

We also compared our data and other recent evidence with clozapine prescribing information issued by regulators in Australia, New Zealand, the US, and the UK.

\section{Methods}

\subsection{Case Identification}

Australian and New Zealand pharmacovigilance data for clozapine-related gastrointestinal complications were obtained from the New Zealand Pharmacovigilance Centre and the Australian TGA.

Our aim was to identify all cases where clozapine was the putative causal agent in serious gastrointestinal hypomotility events. The inclusion criteria were cases coded as: 'serious' or 'life-threatening' constipation, or constipation resulting in hospitalization, surgery or a fatal outcome; ileus; bowel obstruction; bowel ischemia; bowel necrosis; bowel perforation; or megacolon; and where the association with clozapine was considered probable or possible. These criteria are consistent with the internationally recognized definition of a 'serious ADR' [21].

In addition, we sought summary international ADR data on CIGH - held by the WHO in the Uppsala Monitoring Centre, the largest pharmacovigilance databank in the world-for all CIGH reports submitted during 1968-2013 from over 100 countries (including Australia and New Zealand).

We also analyzed official clozapine drug information provided for prescribers and consumers by regulatory bodies in the US (FDA), UK (Medicines \& Healthcare Products Regulatory Agency-the MHRA), New Zealand (Medsafe), and Australia (TGA) to determine the information currently provided on the prevalence, monitoring, and management of CIGH. 


\subsection{Data Extraction}

Pharmacovigilance data were extracted using a pre-specified data extraction form. We obtained country; unique identification number; reported date; reporter location (Australia only); patient demographics including date of birth, gender, height, and weight (Australia only); ADR event characteristics such as date of onset, severity, duration, and outcome; clozapine start date; date of clozapine cessation; clozapine dosage at ADR onset; causality assigned by pharmacovigilance agency; concomitant ADR terms (in accordance with the WHO Adverse Reactions Terminology dictionary); clinical investigations; other medications; treatment; and any additional documented commentary.

Multiple reports of the same (or similar) adverse events for a single patient (identified by demographic data and clinical details) were treated as single cases to eliminate duplications. We excluded reports in which there were confounding comorbid conditions (e.g., bowel cancer, inflammatory bowel disease, hypothyroidism) that may have caused/contributed to the gastrointestinal pathology.

All patients receiving clozapine must be entered into a pharmaceutical registry for monitoring. In order to provide a denominator for estimating $\mathrm{CIGH}$ prevalence, the national registries (operated by Hospira, Douglas, and Novartis) were contacted to calculate the numbers of patients exposed to clozapine over the study period.

\subsection{Ethics Approval}

The pharmacovigilance agencies have been set up to comply with local privacy codes. Their programs have ethics approval and additional approval was not required for identification and analysis of the relevant adverse-effect data.

Identifying demographic data were removed and date of birth was translated into age to preserve anonymity.

\subsection{Data Collection and Analyses}

After data were extracted they were imported into SPSS version 23 (SPSS Inc. Chicago, IL, USA).

Data were analyzed for the following variables: age; gender; clozapine dose at onset of symptoms; duration of clozapine treatment prior to onset of $\mathrm{CIGH}$; symptom onset date; clinical details of presentation, outcome and treatment; and other medications. Descriptive statistics (means $[M]$ with SDs or medians [Md] with interquartile ranges [IQRs]) provide data summaries.

Within the complications group, demographic and clinical covariates were compared between those who were confirmed as having a fatal outcome and those who either recovered or whose outcome was unknown. Continuous variables were compared using $t$ tests for normally distributed variables (e.g., age) or Mann-Whitney $U$ tests for skewed distribution variables (e.g., duration of treatment). Categorical covariates were compared using Pearson Chisquared tests (with Yates' continuity correction for $2 \times 2$ tables).

As a complementary formulation of whether these factors included mortality, several univariate logistic regression models were run to compare fatal and non-fatal outcomes including age (linear variable per 5 years); gender; clozapine dose (linear variable per $100 \mathrm{mg} /$ day); duration of treatment (linear variable per 2 years); and country of reporting. Logistic regression provided odds ratios (ORs) with $95 \%$ confidence intervals adjusted for age, duration of treatment, and country of reporting.

Bivariate correlation was used to describe the change in frequency of CIGH reporting over time.

For hypothesis tests, differences were considered statistically significant when $p<0.05$.

\section{Results}

\subsection{Identification of Cases}

From Australia, there were 6323 clozapine ADR case reports in the TGA 'Gastrointestinal' System Organ Class, of which 157 met the inclusion criteria. A further 33 were excluded (3 duplicates and 30 with confounding pathology). The earliest eligible event occurred in 1994. This resulted in 124 Australian unique reports.

From the New Zealand data, there were 888 clozapine ADR reports in the CARM and IMMP 'Alimentary class', the earliest of which occurred in January 1999, of which 51 met the inclusion criteria. A further 15 were excluded (4 duplicates and 11 with confounding pathology). This resulted in 36 New Zealand unique reports.

Together, this gave a combined series of 160 cases from which data were extracted. Most key variables were reported in over $80 \%$ of cases (e.g., age and gender $94 \%$; duration of treatment $86 \%$; clozapine dose $84 \%$ ), with the notable exception of outcome. Whether the patient ultimately recovered was unknown in $30.6 \%$ of New Zealand cases and $62.9 \%$ of Australian cases. See Box 1 for an example of a case reported to the TGA.

\subsection{Patient Demographics}

Of the 160 patients, $106(66.3 \%)$ were male, 51 female $(31.9 \%)$, and gender was unspecified for three. Age was reported for $150(94 \%)$, with a mean of $46 \pm 13.4$ (SD) years, range $17-76$. 
Box 1 Example of a case reported to the TGA

A 67 -year-old Australian woman had been taking clozapine for 1767 days (almost 5 years) at a dose of 400 mg/day. Other regular medication included nifedipine, quinine sulphate, and estrogen ointment. She presented with abdominal distension and fecal impaction. An abdominal X-ray revealed grossly distended bowel and abdominal distension, with gas and feces in the rectum. She was prescribed laxatives (lactulose and docusate with senna). Two days after onset of symptoms, vomiting with aspiration resulted in cardiorespiratory arrest and death. Ref \#172621

\subsection{Clozapine Treatment}

Clozapine dose was documented for 135 patients $(84 \%)$, $M=439 \pm 221(\mathrm{SD})$, range $=25-1000 \mathrm{mg} /$ day. This is significantly higher than the mean clozapine dose $(372 \mathrm{mg} /$ day $)$ in a large New Zealand epidemiological study [22] $(p<0.0001)$. Clozapine serum levels were seldom available in the ADR reports. The duration of clozapine treatment until serious CIGH, specified for $86 \%$, was usually specific to the day, $\mathrm{Md}=2.5$ years (IQR 4 mo-5 y, 4 mo), range $=3$ days- 18 years. Data were positively skewed (skewness $=1.46, \mathrm{SE}=0.2$ ). Thirty-seven percent of cases occurred within the first year. Duration of treatment until onset of serious CIGH was similar in both Australian and New Zealand populations (see Fig. 1).

\subsection{Concomitant Use of Other Potentially Constipating Medication and of Laxatives}

Only $11 \%$ were recorded as receiving other potentially constipating medications such as those with anticholinergic effects (like benztropine, or tricyclic antidepressants) or other constipating actions (like opioids). From the 70 reports of severe constipation (Table 1), only $6(9 \%)$ were recorded as receiving laxatives prior to the evolution of symptoms.

\subsection{Pathology Reported}

The ADRs reported are summarized in Table 1. Constipation (70 reports) and intestinal obstruction (62 reports) were the most commonly reported features of the CIGH spectrum. A common theme was the late presentation to medical services when significant pathology had already evolved. Although many patients were reported as having massive fecal impaction at autopsy, prior complaints of constipation were not often recorded.

\subsection{Outcome}

Twenty-nine patients were confirmed as having died from suspected CIGH (18\%), 42 were reported as recovered (26\%) and in 89 cases (56\%) the outcome was recorded as 'unknown' or 'not yet recovered' at the time of reporting. The confirmed fatality rates differed significantly between countries: $36.1 \%$ for New Zealand, and $14.8 \%$ for
Fig. 1 Duration of treatment until development of severe clozapine-induced gastrointestinal hypomotility (CIGH) by country

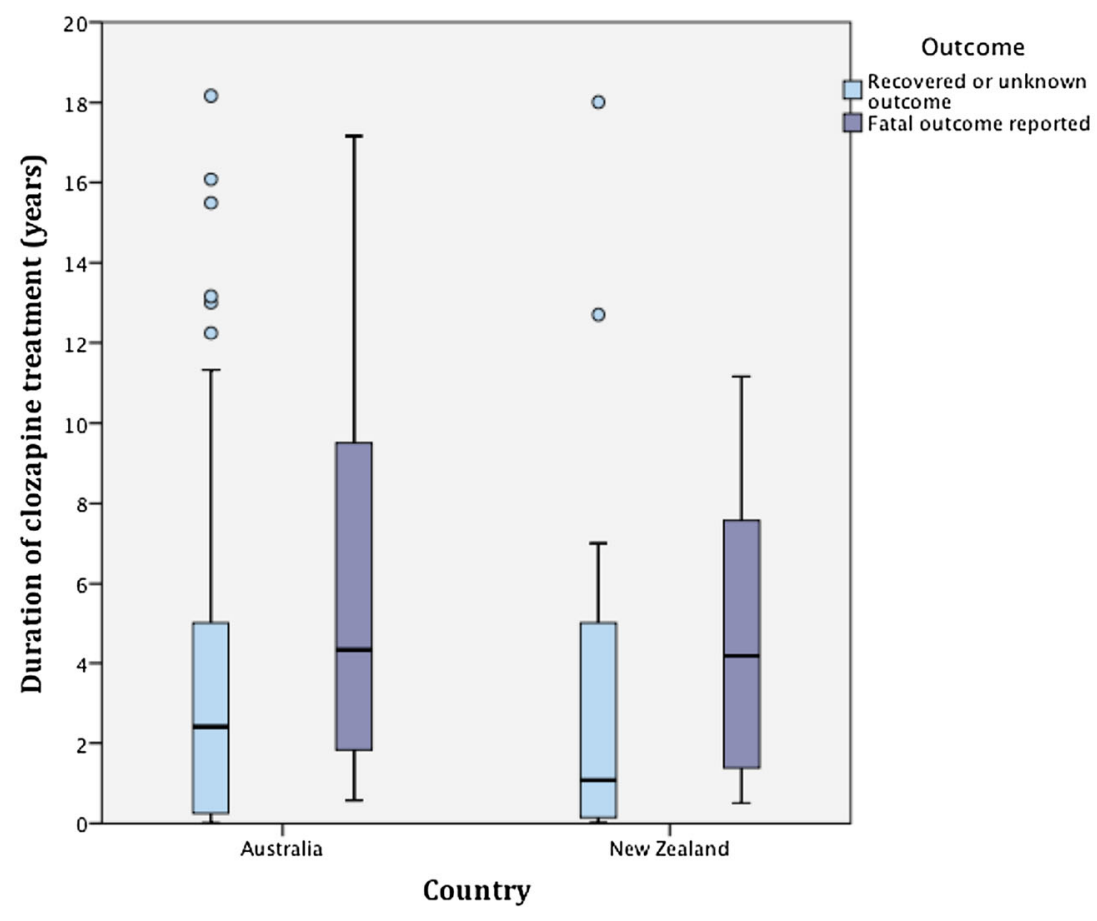


Table 1 Pathology reported in non-fatal and fatal cases of serious $\mathrm{CIGH}$

\begin{tabular}{lcl}
\hline ADR term & Non-fatal cases $[N=131], n(\%)^{\mathrm{a}}$ & Fatal cases $[N=29], n(\%)^{\mathrm{a}}$ \\
\hline Constipation & $62(47.3)$ & $8(27.6)$ \\
Gastrointestinal hypomotility & $4(3.1)$ & $0(0)$ \\
Fecal impaction & $13(9.9)$ & $3(10.3)$ \\
Ileus & $5(3.8)$ & $3(6.9)$ \\
Paralytic ileus & $14(10.7)$ & $1(3.4)$ \\
Ogilvie syndrome & $2(1.5)$ & $0(0)$ \\
Small bowel obstruction & $9(6.9)$ & $1(3.4)$ \\
Pseudo small bowel obstruction & $1(0.8)$ & $0(0)$ \\
Large bowel obstruction & $0(0)$ & $1(3.4)$ \\
Intestinal obstruction & $54(41.2)$ & $8(27.6)$ \\
Intestinal ischemia & $5(3.8)$ & $5(17.2)$ \\
Megacolon & $8(6.1)$ & $3(10.3)$ \\
Bowel perforation & $5(3.8)$ & $6(20.7)$
\end{tabular}

$A D R$ adverse drug reaction, $C I G H$ clozapine-induced gastrointestinal hypomotility

a Total percentages add up to over $100 \%$ as about one third of patients were coded with more than one pathology (for example constipation and intestinal obstruction)
Australia, $\chi^{2}(1, n=160)=8.62, p<0.001$. This is likely to be strongly influenced by missing mortality data; outcomes were confirmed for more New Zealand patients (69.4\%) than Australians (37.1\%), $\chi^{2} \quad(1$, $n=160)=10.55, p<0.001$. This is presumably because while clozapine was under the IMMP in New Zealand, CIGH cases were proactively followed up after initial ADR reporting, leading to better classification of outcomes.

Subgroup analysis was performed comparing patients confirmed as having suffered a fatal outcome with the others. Those with fatal outcomes ranged in age from 22-73 years, with a mean age of 50 years. Unadjusted ORs for the various independent variables are reported in Table 2. A Mann-Whitney $U$ test reveals those with fatal outcomes had significantly longer duration of treatment with clozapine $(\mathrm{Md}=4.2$ years, $\mathrm{IQR}=1.5-8.6$ years $)$ than the rest of the group $(\mathrm{Md}=1.9$ years, $\mathrm{IQR}=0.2-5$ years), $U=968, z=-2.65 p=0.008$ (see Fig. 2). The OR of a fatal outcome increased by $1.21(95 \%$ CI 1.02-1.44) for every 2 years on clozapine. Age, female gender, clozapine dose, and receiving other potentially constipating medication had positive, but non-significant associations with fatal outcomes (Table 2).

Using logistic regression methods to control for confounding, the only variable that remained significant was country: New Zealanders were more likely to have been confirmed as suffering fatal CIGH (OR 4.38, 95\% CI $1.26-15.15)$.

\subsection{Changes in Reporting Over Time}

The number of reported serious CIGH-related drug reactions increased significantly over the 22 -year period. The 3 -year moving average of reported cases increased from below 5 throughout the first decade of clozapine use to consistently above 15 annual cases since 2010 (see Fig. 3). Plotting frequency against date gives a correlation coefficient of $r_{s} 0.9 p<0.001$, indicating a strong positive linear correlation between reporting and time. While CIGH reports increase dramatically, the number of people taking clozapine has also increased. Unfortunately annual use data were not available.
Table 2 Dependent variables and the unadjusted and adjusted odds (adjusted by age, duration of treatment, and country) of a confirmed fatal outcome

\begin{tabular}{lllll}
\hline Variable & Unadjusted OR & $95 \%$ CI & Adjusted OR & 95\% CI \\
\hline Age (per 5-year increment) & 1.16 & $0.99-1.36$ & 1.18 & $0.98-1.42$ \\
Duration of treatment (per 2-year increment) & $1.21^{*}$ & $1.02-1.44$ & 1.16 & $0.95-1.40$ \\
Appearing in NZ dataset & $3.85^{*}$ & $1.61-9.10$ & $4.38^{*}$ & $1.26-15.15$ \\
Dose (per 100-mg/day increment) & 1.07 & $0.88-1.30$ & 1.14 & $0.90-1.44$ \\
Receiving other constipating medication & 2.00 & $0.67-6.25$ & 3.14 & $0.82-12.50$ \\
Gender (being male) & 0.90 & $0.38-2.10$ & 0.85 & $0.39-2.25$ \\
\hline
\end{tabular}

$C I$ confidence interval, $N Z$ New Zealand, $O R$ odds ratio

* Significance at $p<0.05$ 
Fig. 2 Duration of treatment until development of severe clozapine-induced gastrointestinal hypomotility (CIGH) by outcome

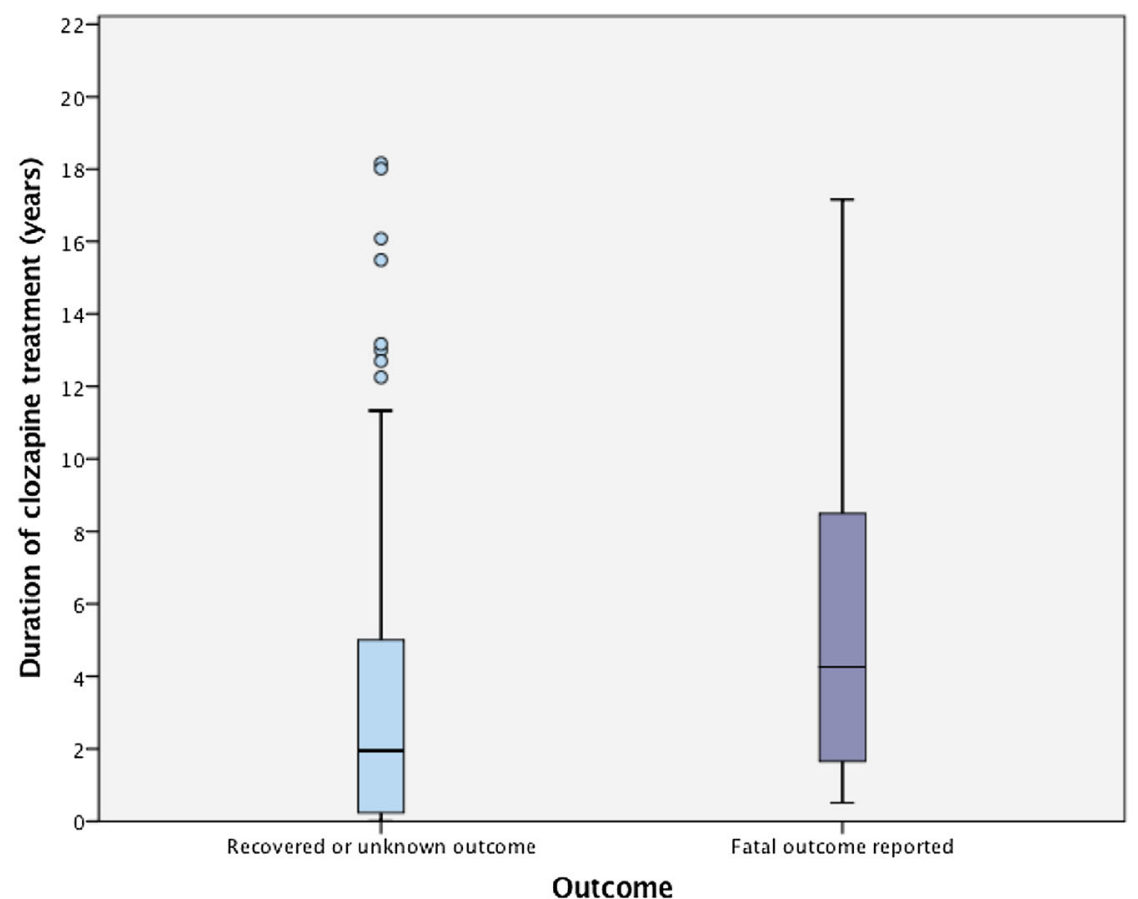

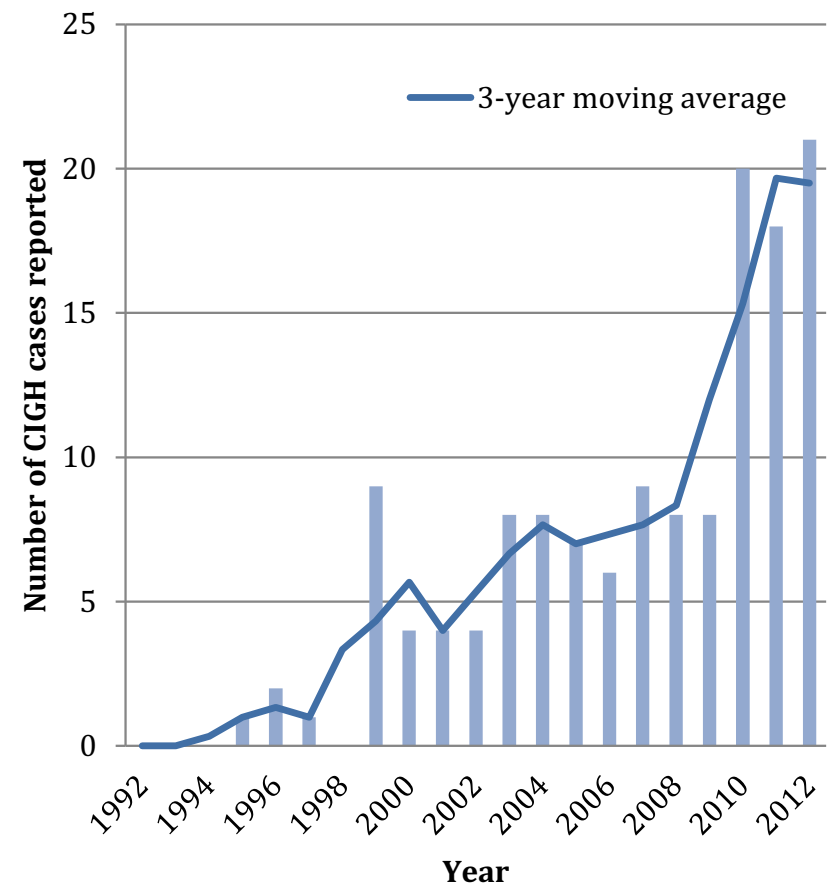

Fig. 3 Severe CIGH pharmacovigilance reports in Australia and New Zealand 1992-2012: total number of cases and 3-year moving average (2013 is not included as full-year data was not collected). $\mathrm{CIGH}$ clozapine-induced gastrointestinal hypomotility

\subsection{Prevalence}

From the time when clozapine first entered the New Zealand market (1988) and mid-2013, 7691 people were exposed to clozapine in New Zealand (personal correspondence: Novartis, New Zealand; and Clopine Services coordinator, Hospira, Australia). Our data show at least 36 of these patients developed serious CIGH, at a prevalence of $0.47 \%$. From the time clozapine first entered the Australian market (1992) to mid-2013, 35,441 people were exposed to clozapine in Australia (personal correspondence: Novartis, Australia; and Clopine Services coordinator, Hospira, Australia). Our data show at least 124 developed serious CIGH, at a prevalence of $0.35 \%$. Although there are limitations in such estimates (as discussed below), this suggests the prevalence of potentially life-threatening CIGH in Australia and New Zealand is at least 37 people for every 10,000 exposed to clozapine.

At least 29 patients died (7/10,000 clozapine users), 16 in Australia and 13 in New Zealand, a reported case fatality rate of $18 \%$. The rates of reported fatal outcomes differed significantly between New Zealand $(17 / 10,000)$ and Australian $(5 / 10,000)$ datasets $\left(\chi^{2}[1, n=43,132]=14.43\right.$, $p<0.001)$. We note that the disproportionate number of Australian patients recorded as 'not yet recovered' or outcome 'unknown' means it is likely some CIGH-related fatal outcomes were not captured, leading to greater underestimation of CIGH fatalities in Australian data.

\subsection{International Pharmacovigilance Data}

The analysis of CIGH-related data from the Uppsala Monitoring Centre is shown in Table 3. The Centre uses a Bayesian confidence neural network to detect ADR signals. 
The information component (IC) is a logarithmic measure of the disproportionality between the observed and the expected reporting of a drug-ADR pair. Signal detection is conducted using the IC025 metric, the lower limit of the $95 \%$ confidence interval for the IC. An IC025 above 0 indicates a statistically significant signal. We report the IC025 metric for associations between clozapine and a number of gastrointestinal symptoms. All the listed gastrointestinal ADR terms in Table 3 have significant positive associations with clozapine. Particularly strong associations exist (IC025 >2) with the terms megacolon, paralytic ileus, and intestinal obstruction.

For comparative purposes, the total number of suspected clozapine-induced agranulocytosis fatalities over the same time period was 168 , with an IC025 of 3.44 .

\subsection{Clozapine Prescribing Information Provided by Regulators}

Information provided by regulators about CIGH was uniformly poor in all four countries reviewed (see Table 4). While information about agranulocytosis, myocarditis, and other adverse effects was prominent and comprehensive (on the first page and under clear and informative headings), no datasheet had a specific section on CIGH, or even referred to this spectrum. Constipation was not mentioned until at least page 6 and was subsumed under an 'anticholinergic side effects' subheading. Serious CIGH conditions like paralytic ileus appeared toward the end of information sheets within long lists of possible side effects, and were reported as being 'very rare', which was defined as "isolated case reports or occurring in less than one in 10,000 users". There was little, if any, reference to CIGH monitoring or treatment.

\section{Discussion}

This is the largest pharmacoepidemiologic study of serious CIGH to date. It includes all 160 Australian and New Zealand patients reported as suffering from serious or lifethreatening CIGH over a 22-year period.

\subsection{Study Limitations}

Retrospective pharmacoepidemiologic studies are subject to a number of limitations.

Prevalence calculations from pharmacoepidemiologic data are compromised by difficulties in determining accurate numerators and denominators. ADR reporting in Australia and New Zealand was, and remains, voluntary and thus does not capture all cases. Spontaneous reporting is dogged by under-reporting [27], with only some $5 \%$ of ADRs reported to pharmacovigilance centers [28]. Although clozapine ADR reporting rates are judged to be high [29] due to obligatory clozapine monitoring systems, reporting is unlikely to be complete. For example, none of the four Dutch patients identified retrospectively by Kok et al. [30] as suffering clozapine-related paralytic ileus had an ADR submitted. Furthermore, spontaneous reporting is vulnerable to selection bias, influenced by the seriousness and novelty of the effect, and by patient characteristics [27]. Serious (and fatal) ADRs are more often reported [31]. These factors mean case fatality rates calculated from such data can be overestimates, while the true prevalence is underestimated. Nonetheless, the Australian data reported here appear to under-report fatalities, as over $60 \%$ of ultimate outcomes were unknown.

Serious CIGH reports increased significantly over the 22-year period. While influenced by the denominator

Table 3 Summary of World Health Organisation data on gastrointestinal adverse drug reactions associated with clozapine

\begin{tabular}{|c|c|c|c|c|}
\hline ADR term & $\begin{array}{l}\text { Total number } \\
\text { of events }(n)\end{array}$ & Fatal outcomes $(F)$ & $\begin{array}{l}\text { Case fatality rate, } \\
\%(F / n \times 100)\end{array}$ & $\mathrm{IC} 025^{\mathrm{a}}$ \\
\hline Megacolon acquired & 56 & 26 & 26 & 3.17 \\
\hline Ileus paralytic & 207 & 48 & 23 & 2.96 \\
\hline Intestinal obstruction & 698 & 177 & 25 & 2.28 \\
\hline Intestinal ischemia & 57 & 39 & 68 & 1.60 \\
\hline Ileus & 154 & 18 & 12 & 1.59 \\
\hline Intestinal gangrene & 24 & 18 & 75 & 1.39 \\
\hline Intestinal necrosis & 17 & 6 & 35 & 1.27 \\
\hline Constipation & 1335 & 178 & 13 & 1.00 \\
\hline Bowel motility disorder & 36 & 6 & 6 & 0.51 \\
\hline Intestinal perforation & 105 & 56 & 53 & 0.51 \\
\hline Dysphagia & 506 & 115 & 23 & 0.37 \\
\hline
\end{tabular}

$A D R$ adverse drug reaction

${ }^{a}$ An IC025 $>0$ indicates a statistically significant signal, an IC025 >2 indicates a particularly strong signal 
Table 4 Review of information regarding CIGH in official clozapine drug information for prescribers issued by regulatory agencies

\begin{tabular}{|c|c|c|c|c|c|c|}
\hline Country & $\begin{array}{l}\text { Is CIGH } \\
\text { spectrum } \\
\text { specifically } \\
\text { described? }\end{array}$ & $\begin{array}{l}\text { Is constipation } \\
\text { identified as a side } \\
\text { effect? }\end{array}$ & $\begin{array}{l}\text { What are the prevalence } \\
\text { estimates of serious CIGH- } \\
\text { type events? }\end{array}$ & $\begin{array}{l}\text { First page } \\
\text { mentioned }\end{array}$ & $\begin{array}{l}\text { Guidance on monitoring } \\
\text { bowel function or treating } \\
\text { CIGH spectrum }\end{array}$ & Source \\
\hline UK & No & $\begin{array}{l}\text { Yes, included } \\
\text { under } \\
\text { anticholinergic } \\
\text { toxicity } \\
\text { subheading, } \\
\text { states rare cases } \\
\text { may be fatal }\end{array}$ & $\begin{array}{l}\text { Intestinal } \\
\text { obstruction/paralytic ileus/ } \\
\text { fecal impaction described as } \\
\text { 'very rare' }\end{array}$ & $\begin{array}{l}\text { Page } 10 \text { for } \\
\text { Clozaril } \\
\text { (constipation } \\
\text { under } \\
\text { anticholinergic } \\
\text { effects) }\end{array}$ & None provided & $\begin{array}{c}\text { MHRA } \\
\text { [23] }\end{array}$ \\
\hline USA & No & $\begin{array}{l}\text { Yes, included } \\
\text { under } \\
\text { anticholinergic } \\
\text { toxicity } \\
\text { subheading, } \\
\text { states rare cases } \\
\text { may be fatal }\end{array}$ & $\begin{array}{l}\text { Fecal impaction, intestinal } \\
\text { obstruction/paralytic ileus } \\
\text { included in long list of } \\
\text { 'adverse events temporally } \\
\text { associated with } \\
\text { clozapine...that may have } \\
\text { no causal relationship with } \\
\text { the drug' }\end{array}$ & $\begin{array}{l}\text { Page } 20 \text { for Fazclo } \\
\text { and page } 17 \text { for } \\
\text { Clozaril } \\
\text { (constipation } \\
\text { under } \\
\text { anticholinergic } \\
\text { effects) }\end{array}$ & $\begin{array}{l}\text { "Constipation should be } \\
\text { initially treated by ensuring } \\
\text { adequate hydration and use } \\
\text { of ancillary therapy such as } \\
\text { bulk laxatives. Consultation } \\
\text { with gastroenterologist is } \\
\text { advisable in more serious } \\
\text { cases" }\end{array}$ & $\begin{array}{l}\text { FDA } \\
{[24]}\end{array}$ \\
\hline Australia & No & $\begin{array}{l}\text { Yes, included } \\
\text { under } \\
\text { anticholinergic } \\
\text { toxicity } \\
\text { subheading, } \\
\text { states rare cases } \\
\text { may be fatal }\end{array}$ & $\begin{array}{l}\text { Ileus and impaction } \\
\text { categorized as 'rare' } \\
\text { Intestinal obstruction/fecal } \\
\text { impaction described as 'very } \\
\text { rare' }(<1 / 10,000 \text { including } \\
\text { isolated case reports })\end{array}$ & $\begin{array}{l}\text { Page } 6 \\
\text { (constipation } \\
\text { under } \\
\text { anticholinergic } \\
\text { effects) }\end{array}$ & None provided & $\begin{array}{c}\text { TGA } \\
{[25]}\end{array}$ \\
\hline $\begin{array}{l}\text { New } \\
\text { Zealand }\end{array}$ & No & $\begin{array}{l}\text { Yes, included } \\
\text { under } \\
\text { anticholinergic } \\
\text { toxicity } \\
\text { subheading, } \\
\text { states rare cases } \\
\text { may be fatal }\end{array}$ & $\begin{array}{l}\text { Intestinal obstruction/ileus/ } \\
\text { fecal impaction described as } \\
\text { 'very rare' }(<1 / 10,000 \\
\text { including isolated case } \\
\text { reports })\end{array}$ & $\begin{array}{l}\text { Page } 9 \\
\text { (constipation } \\
\text { under } \\
\text { anticholinergic } \\
\text { effects) }\end{array}$ & $\begin{array}{l}\text { "Patients should be } \\
\text { questioned about their } \\
\text { bowel habits" }\end{array}$ & $\begin{array}{c}\text { Medsafe } \\
\text { [26] }\end{array}$ \\
\hline
\end{tabular}

$C I G H$ clozapine-induced gastrointestinal hypomotility, FDA US Food and Drug Administration, MHRA UK Medicines and Healthcare Products Regulatory Agency, TGA Australian Therapeutic Goods Administration

(number of people receiving clozapine) increasing, growing awareness of the relationship between clozapine and gastrointestinal hypomotility is also likely to have improved reporting over time. No reports of CIGH appeared in the databases until 1994 in Australia and 1999 in New Zealand, 2 and 11 respective years after clozapine became available. Perhaps earlier events were not attributed to clozapine and consequently were not reported as ADRs. If so, our prevalence estimate is further understated.

Finally, pharmacovigilance data, while potentially powerful in identifying possible adverse reactions through association, cannot prove causation.

\subsection{Implications of Results}

The results demonstrate that there is no consistent relationship between age, dose, or duration of treatment and the onset of life-threatening CIGH. Mean clozapine doses in those with serious CIGH were higher than in the general population but, as evidenced by the wide dose range, serious CIGH also occurred at low clozapine doses. Serum clozapine levels were not often available in ADR reporting, so we could not test whether higher serum levels increased the risk for CIGH, as previously reported [6]. Although more men were affected than women, this reflects the demographics of the treatment population (e.g., approximately two-thirds of Australian clozapine-treated patients are male) [32]. Unfortunately this means it is hard to predict which clozapine-treated patients are most at risk of serious CIGH.

It could be argued that association alone should not 'incriminate' clozapine. However, the strength of the association in our Australian and New Zealand data is also reflected in the WHO pharmacovigilance database, with a strong positive correlation (IC025 >2) between clozapine and megacolon, ileus, and intestinal ischemia. 
Constipation's IC025 of 1 is lower than expected, given its established prevalence of around $30-60 \%$ in clozapinetreated patients [33-36]. This probably reflects the perception that constipation is a relatively benign adverse effect, with reporting to pharmacovigilance agencies only occurring in extreme cases, as indicated by the constipation fatality rate of $13 \%$ in the $\mathrm{WHO}$ data. Internationally, constipation is now implicated in more clozapine-related deaths than agranulocytosis (178 compared with 168).

It is noteworthy that agranulocytosis is regularly and compulsorily checked through blood films, enabling complete identification of all cases and high levels of reporting, and is coded under simple ADR terminology. We hypothesize that the rate of serious $\mathrm{CIGH}$ has been underestimated due to its many pathological synonyms and related terms - essentially 'splitting the vote'-which has weakened the pharmacovigilance signal, in contrast to the single term for agranulocytosis. Nonetheless, the IC025s of megacolon and paralytic ileus alone are very similar to the IC025 of agranulocytosis.

Clozapine drug safety information emphasizes the risk of agranulocytosis in 'black box' warnings and provides comprehensive guidance on management. Mortality rates related to agranulocytosis have been falling due to careful vigilance, early detection, and better treatment, with a recent study finding fatalities averaged 0.45 (95\% CI 0.00-0.92) for 10,000 treated individuals/year [37]. Conversely, both the reported incidence and mortality rates of $\mathrm{CIGH}$ have been rising. Deaths from CIGH now far exceed those from agranulocytosis-in our study $\mathrm{CIGH}$ fatalities occurred in 7/10,000 clozapine users, a likely underestimation. While growing awareness of the CIGH spectrum may have improved case reporting, it should have also increased awareness of the need for preventative treatment.

While clozapine-related myocarditis and agranulocytosis occur early in treatment, warranting greater vigilance during clozapine initiation [14], the wide SD of time-toonset of $\mathrm{CIGH}$ indicates it can occur at any stage of treatment. Patients with serious CIGH often presented late, with advanced pathology. Although many patients were reported as having massive fecal impaction at autopsy, prior complaints of constipation had not often been recorded. Among those with constipation, only $9 \%$ were noted as having been prescribed laxatives prior to development of the serious ADR. This suggests constipation is not being recognized and treated seriously, despite the sometimes fatal consequences. This is consistent with other reports of low subjective awareness of objective hypomotility [5, 6, 38] and reinforces our earlier observation that clozapine-treated patients may not recognize or experience constipation in the same way as others due to changes in pain sensitivity or habituation, and thus may be less likely to complain $[6,38]$. This decreased sensitivity places the responsibility for actively monitoring and treating $\mathrm{CIGH}$ on health professionals; yet the potential severity of CIGH remains poorly recognized amongst mental health staff [8, 15, 17, 39-41].

\subsection{Recommendations for Regulators}

We recommend regulators update their guidance on clozapine to reflect current knowledge about CIGH.

Recent literature emphasizes the need for better understanding and education regarding CIGH [40, 42]. Regulatory agencies and pharmaceutical companies share some responsibility for this. Official drug safety information is currently inadequate. It does not highlight the CIGH condition and underestimates both severity and prevalence. Our study and others find 40-fold higher rates of serious $\mathrm{CIGH}$ than those indicated by regulators, with the cumulative incidence in other smaller studies also varying between 20-90/10,000 [4, 12, 13, 43], despite most studies investigating just one subset of the $\mathrm{CIGH}$ spectrum such as subileus [43], ileus [13], or bowel obstruction [4], hence under-estimating combined rates.

The deficiencies in drug safety information likely contribute to poor prevention and treatment of $\mathrm{CIGH}$, delayed recognition and elevated morbidity and mortality rates. There has been sufficient evidence published over the last 15 years to warrant regulators reviewing and updating the information they provide prescribers and consumers on CIGH.

\subsection{Recommendations for Health Professionals, Carers and Clozapine Users}

We recommend careful monitoring of bowel function and the use of prophylactic laxatives, for all clozapine users.

Few patients in this study were treated with laxatives prior to developing CIGH. We have previously argued there should be a strong focus on preventing and managing CIGH [38, 44] in all clozapine-treated patients, even those not subjectively reporting $\mathrm{CIGH}$ symptoms. Assertive monitoring and treatment of $\mathrm{CIGH}$ is recommended by Flanagan and Ronaldson [40] and by the Maudsley Prescribing Guidelines [45]. The body of evidence on which treatment to select is not strong. A Cochrane review on pharmacological treatment of antipsychotic-related constipation identified only a handful of eligible trials that considered the efficacy of laxatives in this population, with all trials of poor quality [42].

In an intervention study, when docusate and senna augmented by macrogol was prescribed to clozapine-treated patients according to the Porirua Protocol (available at http://hdl.handle.net/10523/6763), gastrointestinal transit times improved significantly [38]. Before the Protocol was 
introduced, the prevalence of serious CIGH was 8.2 cases per 100 person-years, reducing to 1.1 cases per 100 personyears in the 5 years after implementation $(p<0.001)$ [38]. Another study similarly showed that the pre-emptive use of macrogol in psychiatric intensive care was well tolerated and reduced the incidence of severe constipation, especially in patients receiving clozapine and zuclopenthixol [46]. These findings suggest intervention is possible and effective.

Alongside prophylactic laxatives, we suggest careful monitoring of bowel function and a high index of suspicion for gastrointestinal symptoms and signs that herald emerging serious bowel pathology - the 'red flags' on the Porirua Protocol [38]. These include either moderate to severe abdominal pain lasting over an hour; or any abdominal pain/discomfort lasting over an hour and any one or more of the following: abdominal distension; diarrhea; vomiting; absent or high-pitched bowel sounds; metabolic acidosis; hemodynamic instability; leukocytosis; or other signs of sepsis. These signs and symptoms must be treated seriously and an urgent medical and/or surgical opinion sought, notifying the assessing clinician that the person is taking clozapine and consequently at high risk for serious gastrointestinal complications.

\section{Conclusions}

Over the last decade, clozapine has been increasingly recognized as causing gastrointestinal hypomotility or 'slow gut'. In our study, the reported prevalence of serious CIGH was $37 / 10,000$, a likely underestimation of true prevalence. It appears anyone on clozapine may develop CIGH; we identified no clear demographic or pharmacologic risk factors.

While clozapine is undoubtedly the best treatment for many, its use requires careful management due to its adverse effects. Considerable resources are devoted to monitoring for agranulocytosis in clozapine-treated patients, but more patients die from CIGH than from agranulocytosis. Efforts to address $\mathrm{CIGH}$ at a regulatory or clinical level have been limited, at best.

Current clozapine prescribing information provided by regulators provides inadequate information about CIGH. This may be contributing to poor awareness of the CIGH spectrum and high morbidity and mortality rates. It is time this guidance is updated to reflect current knowledge about CIGH.

Serious CIGH is a medical emergency. Assertive monitoring and early detection of clozapine-related agranulocytosis have greatly reduced mortality rates; similar attention needs to be given to CIGH.
Acknowledgements We acknowledge the TGA and the New Zealand Pharmacovigilance Centre for their significant assistance, especially Janelle Ashton. Thanks to James Stanley, biostatistician, for advice on statistical analyses.

\section{Compliance with ethical standards}

Funding This study received no external funding. The open access fee was paid for by Capital and Coast District Health Board continuing medical education funds.

Conflict of interest Susanna Every-Palmer and Pete Ellis have declared that there are no conflicts of interest in relation to the subject of this study.

Open Access This article is distributed under the terms of the Creative Commons Attribution-NonCommercial 4.0 International License (http://creativecommons.org/licenses/by-nc/4.0/), which permits any noncommercial use, distribution, and reproduction in any medium, provided you give appropriate credit to the original author(s) and the source, provide a link to the Creative Commons license, and indicate if changes were made.

\section{References}

1. Taylor DM. Clozapine for treatment-resistant schizophrenia: still the gold standard? CNS Drugs. 2017;31(3):4.

2. Meltzer HY. Treatment-resistant schizophrenia-the role of clozapine. Curr Med Res Opin. 1997;14(1):1-20.

3. Tiihonen J, Lönnqvist J, Wahlbeck K, Klaukka T, Niskanen L, Tanskanen A, et al. 11-year follow-up of mortality in patients with schizophrenia: a population-based cohort study (FIN11 study). Lancet. 2009;374(9690):620-7.

4. Stroup T, Gerhard T, Crystal S, Huang C, Olfson M. Comparative effectiveness of clozapine and standard antipsychotic treatment in adults with schizophrenia. Am J Psychiatry. 2016;173(2):166.

5. Baptista T, Carrizo E, Fernandez E, Connell L, Servigna M, Parra A, et al. Colonic transit diagnostic test shows significant gastrointestinal hypomotility in clozapine-treated patients in comparison with subjects treated with other antipsychotics. Schizophr Res. 2015;166:4.

6. Every-Palmer S, Nowitz M, Stanley J, Grant E, Mark H, Dunn H, et al. Clozapine-treated patients have marked gastrointestinal hypomotility, the probable basis of life-threatening gastrointestinal complications: a cross sectional study. EBioMedicine. 2016;5:125-34.

7. Every-Palmer S, Lentle RG, Reynolds G, Hulls C, Chambers P, Dunn $\mathrm{H}$, et al. Spatiotemporal mapping techniques show clozapine impairs neurogenic and myogenic patterns in the colon of the rabbit in a dose-dependent manner. Front Pharmacol. 2017:8:209.

8. Shirazi A, Stubbs B, Gomez L, Moore S, Gaughran F, Flanagan $\mathrm{RJ}$, et al. Prevalence and predictors of clozapine-associated constipation: a systematic review and meta-analysis. Int $\mathrm{J}$ Mol Sci. 2016;17(6):863.

9. Dennison C, Prasad M, Lloyd A, Bhattacharyya SK, Dhawan R, Coyne $\mathrm{K}$. The health-related quality of life and economic burden of constipation. Pharmacoeconomics. 2005;23(5):461-76.

10. Damon H, Dumas P, Mion F. Impact of anal incontinence and chronic constipation on quality of life. Gastroenterol Clin Biol. 2004;28(1):16-20.

11. Wald A, Scarpignato C, Kamm M, Mueller-Lissner S, Helfrich I, Schuijt $\mathrm{C}$, et al. The burden of constipation on quality of life: 
results of a multinational survey. Aliment Pharmacol Ther. 2007;26(2):227-36.

12. Palmer SE, McLean RM, Ellis PM, Harrison-Woolrych M. Lifethreatening clozapine-induced gastrointestinal hypomotility: an analysis of 102 cases. J Clin Psychiatry. 2008;69(5):759-68.

13. Nielsen J, Meyer JM. Risk factors for ileus in patients with schizophrenia. Schizophr Bull. 2012;38(3):592-8.

14. Cohen D, Bogers JP, van Dijk D, Bakker B, Schulte PF. Beyond white blood cell monitoring: screening in the initial phase of clozapine therapy. J Clin Psychiatry. 2012;73(10):1307-12.

15. De Hert M, De Beugher A, Sweers K, Wampers M, Correll CU, Cohen D. Knowledge of psychiatric nurses about the potentially lethal side-effects of clozapine. Arch Psychiatr Nurs. 2016;30(1):79-83.

16. Ally BA, Stallman HM. Evaluation of a clozapine decision support tool in a mental health facility. J Pharm Pract Res. 2016;46(2):137-8.

17. Nielsen J, Young C, Ifteni P, Kishimoto T, Xiang Y-T, Schulte $\mathrm{PF}$, et al. Worldwide differences in regulations of clozapine use. CNS Drugs. 2016;30(2):149-61.

18. World Health Organization. Uppsala Report 61. 2013. http:// www.who-umc.org/media/1681/27577.pdf. Accessed 9 June 2017.

19. Coulter DM. The New Zealand intensive medicines monitoring programme in pro-active safety surveillance. Pharmacoepidemiol Drug Saf. 2000;9(4):273-80.

20. Meyboom R, Hekster Y, Egberts A, Gribnau F, Edwards I. Causal or casual? The role of causality assessment in pharmacovigilance. Drug Saf. 1997;17(6):374.

21. Council for International Organization of Medical Science (CIOMS). Current challenges in pharmacovigilance: pragmatic approaches (report of CIOMS working group V). Geneva; 2001.

22. Wheeler AJ. Treatment pathway and patterns of clozapine prescribing for schizophrenia in New Zealand. Ann Pharmacother. 2008;42(6):852-60.

23. Medicines \& Healthcare Products Regulatory Agency. http:// www.mhra.gov.uk/spc-pil/index.htm. Accessed 9 June 2017.

24. Food and Drug Administration. https://www.accessdata.fda.gov/ scripts/cder/daf/. Accessed 9 June 2017.

25. Therapeutic Goods Administration. https://www.tga.gov.au/ product-information-pi. Accessed 9 June 2017.

26. Medsafe. http://www.medsafe.govt.nz/Medicines/infoSearch.asp. Accessed 9 June 2017

27. Moride Y, Haramburu F, Requejo AA, Begaud B. Under-reporting of adverse drug reactions in general practice. Br J Clin Pharmacol. 1997;43(2):177.

28. Hazell L, Shakir SA. Under-reporting of adverse drug reactions. Drug Saf. 2006;29(5):385-96.

29. Hill GR, Harrison-Woolrych M. Clozapine and myocarditis: a case series from the New Zealand Intensive Medicines Monitoring Programme. N Z Med J. 2008;121(1283):68-75.

30. Kok JT, van der Heijden FMMA, et al. Ileus tijdens onderhoudsbehandeling met clozapine [Dutch]. Pharmaceutisch Weekblad/Wetenschappelijk Platform. 2007;1(1):2.
31. Lumley C, Walker S, Hall G, Staunton N, Grob P. The underreporting of adverse drug reactions seen in general practice. Pharm Med. 1986;1(3):205-12.

32. Haas SJ, Hill R, Krum H, Liew D, Tonkin A, Demos L, et al. Clozapine-associated myocarditis. Drug Saf. 2007;30(1):47-57.

33. Hayes G, Gibler B. Clozapine-induced constipation. Am J Psychiatry. 1995;152(2):298.

34. Lieberman JA, Safferman AZ, Pollack S, Szymanski S, Johns C, Howard A, et al. Clinical effects of clozapine in chronic schizophrenia: response to treatment and predictors of outcome. Am J Psychiatry. 1994;151(12):1744-52.

35. Miller D. Review and management of clozapine side effects. J Clin Psychiatry. 2000;61:14.

36. Antonacci DJ, De Groot C. Clozapine treatment in a population of adults with mental retardation. J Clin Psychiatry. 2000;61(1):22-5.

37. Balda MV, Garay OU, Papale RM, Bignone I, Bologna VG, Brandolini A, et al. Clozapine-associated neutropenia and agranulocytosis in Argentina (2007-2012). Int Clin Psychopharmacol. 2015;30(2):109-14.

38. Every-Palmer S, Ellis PM, Nowitz M, Stanley J, Grant E, Huthwaite $\mathrm{M}$, et al. The porirua protocol in the treatment of clozapine-induced gastrointestinal hypomotility and constipation: a pre-and post-treatment study. CNS Drugs. 2017;31(1):75-85.

39. Mohan I, Wendelborn K. Constipation associated with clozapine: need for closer monitoring. Australas Psychiatry. 2016;24(1):97.

40. Flanagan RJ, Ronaldson KJ. Gastrointestinal hypomotility and dysphagia. In: Manu P, Flanagan RJ, Ronaldson KJ, editors. Lifethreatening effects of antipsychotic drugs. London: Academic Press; 2016.

41. Flanagan RJ, Ball RY. Gastrointestinal hypomotility: an underrecognised life-threatening adverse effect of clozapine. Forensic Sci Int. 2011;206(1-3):e31-6.

42. Every-Palmer S, Newton-Howes G, Clarke MJ. Pharmacological treatment for antipsychotic-related constipation. Cochrane Database Syst Rev. 2017;1:CD011128. doi:10.1002/14651858. CD011128.pub2.

43. Grohmann R, Rüther E, Sassim N, Schmidt L. Adverse effects of clozapine. Psychopharmacology (Berlin). 1989;99(1):S101-4.

44. Every-Palmer S, Ellis PM, Flanagan RJ, Baptista T. Mild to moderate clozapine-induced gastrointestinal hypomotility should not require cessation of clozapine. Gen Hosp Psychiatry. 2017 (in press).

45. Taylor D, Paton C, Kapur S. The Maudsley prescribing guidelines in psychiatry. New York: Wiley; 2015.

46. Bulot V, Lemogne C, Nebot N, Blondon H, Roux P. Systematic prevention of severe constipation induced by antipsychotic agents: a quasi-experimental study. Eur Neuropsychopharmacol. 2016;26(10):1690-1. 